\title{
Measurements of heavy-flavour decay leptons with ALICE
}

\author{
Shingo Sakai ${ }^{1, a}$, on behalf of the ALICE Collaboration \\ ${ }^{1}$ Istituto Nazionale di Fisica Nucleare - LNF via E.Fermi, 40 - 00044 Frascati (Rome) - Italy
}

\begin{abstract}
We present measurements of electrons and muons from heavy-flavour hadron decays at central and forward rapidity performed by the ALICE Collaboration in $\mathrm{p}-\mathrm{Pb}$ $\left(\sqrt{s_{\mathrm{NN}}}=5.02 \mathrm{TeV}\right)$ and $\mathrm{Pb}-\mathrm{Pb}$ collisions $\left(\sqrt{s_{\mathrm{NN}}}=2.76 \mathrm{TeV}\right)$. Electrons are reconstructed using several detectors of the ALICE central barrel. Muons are reconstructed using the muon spectrometer at forward rapidity $(2.5<y<4)$. The nuclear modification factors in $\mathrm{Pb}-\mathrm{Pb}\left(R_{\mathrm{AA}}\right)$ and in $\mathrm{p}-\mathrm{Pb}\left(R_{\mathrm{pPb}}\right)$ collisions, and the azimuthal anisotropy $\left(v_{2}\right)$ in $\mathrm{Pb}-$ $\mathrm{Pb}$ collisions will be discussed. Theoretical predictions are compared with the data. In addition, the measurement of the azimuthal correlation between electrons from heavyflavour hadron decays and charged hadrons in $\mathrm{p}-\mathrm{Pb}$ collisions will be shown.
\end{abstract}

\section{Introduction}

ALICE (A Large Ion Collider Experiment) is the experiment dedicated to the study of ultra-relativistic heavy-ion collisions at the LHC. In such collisions, hot and dense QCD matter is formed. Heavy quarks, i.e. charm and beauty, are produced primarily in hard partonic scattering processes occurring in the initial stage of the collision. In pp collisions, their production is well described by perturbative QCD (pQCD) calculations. In heavy-ion collisions, heavy quarks propagate through and interact with the hot and dense QCD matter. Therefore, measurements of heavy-flavour production provide relevant information on the properties of the medium created in the collisions. A strong suppression of heavy-flavour production has been observed at high transverse momentum $\left(p_{\mathrm{T}}\right)$ with respect to the cross section measured in pp collisions and scaled by the number of nucleon-nucleon collisions $[1,2]$, and it is mainly interpreted in terms of energy loss of heavy quarks in the QCD matter via both elastic and inelastic interactions with the medium constituents. Based on model calculations, the energy loss of heavy quarks is expected to be smaller than for light quarks. Furthermore, the energy loss depends on the colour charge of the parton. The theoretical models predict a mass hierarchy in partonic energy loss, $\Delta E_{\mathrm{g}}>\Delta E_{\text {light-q }}>\Delta E_{\mathrm{c}}>\Delta E_{\mathrm{b}}$ [3]. Experimentally the mass hierarchy can be addressed by measuring the nuclear modification factors of different hadron species. Further properties of the medium can be studied by measuring the azimuthal anisotropy of heavy-flavour production in non-central collisions, particularly the elliptic flow $\left(v_{2}\right)$ that is defined by the second harmonic of the Fourier expansion of the particle-momentum azimuthal distribution with respect to the orientation of the reaction plane. The measurement of $v_{2}$ at low $p_{\mathrm{T}}$ is sensitive to the coupling of heavy quarks with the medium and possibly their thermalization in the medium. At high $p_{\mathrm{T}}, v_{2}$

\footnotetext{
a e-mail: Shingo.Sakai@lnf.infn.it
} 
reflects the path-length dependence of energy loss due to the initial spatial anisotropy in non-central collisions.

Cold nuclear matter effects influencing heavy-flavour production in heavy-ion collisions, such as modification of Parton Distribution Functions (PDFs) in nuclei (shadowing/saturation at small Bjorken- $x$ ) and $k_{\mathrm{T}}$ broadening $[4,5]$, can be studied in $\mathrm{p}-\mathrm{A}$ collisions in which the hot and dense $\mathrm{QCD}$ medium is not expected to be formed. One of the most interesting results in $\mathrm{p}-\mathrm{Pb}$ collisions was the observation in high multiplicity events of two long-range ridge-like structures in the distributions of angular correlations between two charged particles after removing correlations due to jets as extracted from low-multiplicity collisions [6-8]. This double-ridge structure can be interpreted as due to a collective expansion of the final-state particles or to gluon saturation effects in the initial state in the Colour Glass Condensate (GCG) framework.

Measuring the two-particle angular correlations using a heavy-flavour decay electron as trigger particle can be useful to further understand the emergence of the two-ridge structure. In these proceedings we present ALICE measurements of electrons and muons from semi-leptonic decays of open charm and beauty hadrons at mid and forward rapidity in $\mathrm{Pb}-\mathrm{Pb}$ collisions at $\sqrt{s_{\mathrm{NN}}}=2.76 \mathrm{TeV}$ reporting the nuclear modification factor $\left(R_{\mathrm{AA}}\right)$ and $v_{2}$. These results are discussed together with the measurements in $\mathrm{p}-\mathrm{Pb}$ collisions at $\sqrt{s_{\mathrm{NN}}}=5.02 \mathrm{TeV}$, and they are compared with predictions from several theoretical models that calculate both $R_{A A}$ and $v_{2}$. Azimuthal correlations between electrons from heavy-flavour hadron decays and charged hadrons in $\mathrm{p}-\mathrm{Pb}$ collisions are shown as well.

\section{Analysis}

The results discussed in these proceedings are obtained from the 2010 and 2011 LHC runs with $\mathrm{Pb}-$ $\mathrm{Pb}$ collisions at $\sqrt{s_{\mathrm{NN}}}=2.76 \mathrm{TeV}$, and from the $2013 \mathrm{LHC}$ run with $\mathrm{p}-\mathrm{Pb}$ collisions at $\sqrt{s_{\mathrm{NN}}}=5.02$ $\mathrm{TeV}$. The interaction trigger was based on the VZERO detector, which is composed of two arrays of scintillator tiles covering the full azimuth in the pseudorapidity regions $2.8<\eta<5.1$ (VZERO-A) and $-3.7<\eta<-1.7$ (VZERO-C). The VZERO detector also contributes to the collision centrality determination which is expressed in terms of percentiles of the hadronic $\mathrm{Pb}-\mathrm{Pb}$ cross section, and to estimation of the reaction plane, which is defined by the impact parameter and the beam axis, for the elliptic-flow measurement. In $\mathrm{Pb}-\mathrm{Pb}$ events, data collected with an interaction trigger based on the VZERO detector and enriched with central (0-10\%) and semi-central (10-50\%) events via a selection on the VZERO signal amplitude were analyzed. In addition, events triggered with the ElectroMagnetic Calorimeter (EMCal), which covers an azimuthal angle $\Delta \varphi=100^{\circ}$ in $|\eta|<0.7$, were used to extend the $p_{\mathrm{T}}$ reach of the electron measurements. The EMCal trigger requires an electromagnetic shower with energy above given a threshold, with the shower defined by a sliding window of $4 \times 4$ EMCal cells (corresponding to $0.056 \times 0.056$ in $\Delta \varphi \times \Delta \eta$ ).

Electron identification was carried out by combining information from several detectors. A set of reconstructed tracks in the TPC (Time Projection Chamber) and in the Inner Tracking System (ITS) was selected with at least one associated hit in the Silicon Pixel Detector (SPD) to reduce conversions from outside of the SPD which is the innermost part of the ITS, and with the $\mathrm{d} E / \mathrm{d} x$ in the TPC gas corresponding to the expectation for an electron to suppress hadron contamination in the electron sample. Additional hadron rejection utilized the time-of-flight measured with the TOF detector at low $p_{\mathrm{T}}$ and the energy deposited in the EMCal. The ratio of the energy measured with the calorimeter $(E)$ and the momentum measured with the TPC $(p)$ is close to unity for an electron $(E / p \sim 1)$, while the ratio for a charged hadron is much smaller on average. Electrons from heavy-flavour hadron decays are statistically separated from the background, which is mainly from Dalitz decays of neutral mesons and photon conversions in the detector material (photonic electrons). The background from photonic 
electrons is estimated with two different methods. In the first case, the yield of photonic electrons is measured by pairing electrons with oppositely charged partner tracks and requiring the invariant mass of the $\mathrm{e}^{+} \mathrm{e}^{-}$pair to be close to zero. In the second case, the background is calculated with a Monte Carlo cocktail based on the measured distributions of $\pi^{0}$ and contribution from other background electron sources from neutral mesons.

Muons at forward rapidity were measured with the muon tracking and trigger chambers. A muon track candidate was required to be matched with the corresponding track measured in the trigger chambers, which, being downstream of an iron wall, provide an efficient rejection of hadrons that cross the main absorber and the tracking chambers. The remaining background, mainly due to muons from pions and kaons that decay before the absorber, was evaluated using a Monte Carlo simulation for $\mathrm{pp}$ collisions, and using a data driven approach in the $\mathrm{p}-\mathrm{Pb}$ and $\mathrm{Pb}-\mathrm{Pb}$. In the latter, the $p_{\mathrm{T}}$ distributions of pions and kaons measured at mid-rapidity are extrapolated to forward rapidity where muons are measured. Then the decay-muon $p_{\mathrm{T}}$ distribution was calculated via a simulation [13]. The signal of heavy-flavour decay muons was obtained by subtracting the background component from the measured inclusive spectrum.

\section{Results and discussion}

The nuclear modification factors of heavy-flavour decay electrons $(|y|<0.6)$ and muons $(2.5<y<4)$ measured in $\mathrm{Pb}-\mathrm{Pb}$ collisions for two centrality classes, most central ( $0-10 \%$ centrality, left) and midcentral (40-50\% for electrons and 40-80\% centrality for muons, right), are shown in Fig.1 as a function of $p_{\mathrm{T}}$. The nuclear modification factor is defined as:

$$
R_{\mathrm{AA}}=\frac{\mathrm{d} N_{\mathrm{AA}} / \mathrm{d} p_{\mathrm{T}}}{\left\langle T_{\mathrm{AA}}\right\rangle \mathrm{d} \sigma_{\mathrm{pp}} / \mathrm{d} p_{\mathrm{T}}},
$$

where $\left\langle T_{\mathrm{AA}}\right\rangle$ is the nuclear overlap function for the considered centrality interval obtained via Glauber MC calculations [9], $\mathrm{d} N_{\mathrm{AA}} / \mathrm{d} p_{\mathrm{T}}$ is the $p_{\mathrm{T}}$-differential yield in $\mathrm{Pb}-\mathrm{Pb}$ collisions and $\mathrm{d} \sigma_{\mathrm{pp}} / \mathrm{d} p_{\mathrm{T}}$ is the $p_{\mathrm{T}}$-differential cross section in pp collisions. The cross section in pp collisions was obtained by scaling the cross sections measured at $\sqrt{s}=7 \mathrm{TeV}$ [10] down to $2.76 \mathrm{TeV}$ according to the $\sqrt{s}$ dependence predicted by FONLL [11] for electrons from heavy-flavour hadron decays. Due to the limited $p_{\mathrm{T}}$ range for the electron measurement in pp collisions, the FONLL calculation for $\sqrt{s}=2.76 \mathrm{TeV}$ [12] was used as the reference for $p_{\mathrm{T}}>8 \mathrm{GeV} / c$. For the muons, the cross section measured in $\mathrm{pp}$ collisions at $\sqrt{s}=2.76 \mathrm{TeV}$ could be used directly as reference [13]. In central $\mathrm{Pb}-\mathrm{Pb}$ collisions, the yields of electrons at mid rapidity and muons at forward rapidity from heavy-flavour hadron decays are strongly suppressed at $p_{\mathrm{T}}>3 \mathrm{GeV} / c$ with respect to the binary-collisions scaled cross section in pp collisions at the same energy. The results indicate energy loss of heavy quarks in the medium. The magnitude of suppression due to the energy loss is similar in the different rapidity regions within statistical and systematic uncertainties. The magnitude of suppression is centrality dependent, and the suppression is smaller in mid-central compared to central $\mathrm{Pb}-\mathrm{Pb}$ collisions both for electrons and muons. To investigate the quark-mass dependence of parton energy loss, it is crucial to measure electrons from beauty hadron decays in $\mathrm{pp}$ and $\mathrm{Pb}-\mathrm{Pb}$ collisions. Figure 2 shows the $R_{\mathrm{AA}}$ of electrons from beauty-hadron decays in the $20 \%$ most central $\mathrm{Pb}-\mathrm{Pb}$ collisions. The fraction of electrons from beauty-hadron decays was extracted based on the electron impact parameter distribution by comparing templets obtained from simulations. Within the current uncertainties, there is a hint of suppression of electrons from beauty decays for $p_{\mathrm{T}}>3 \mathrm{GeV} / c$ in the $20 \%$ most central $\mathrm{Pb}-\mathrm{Pb}$ collisions. 

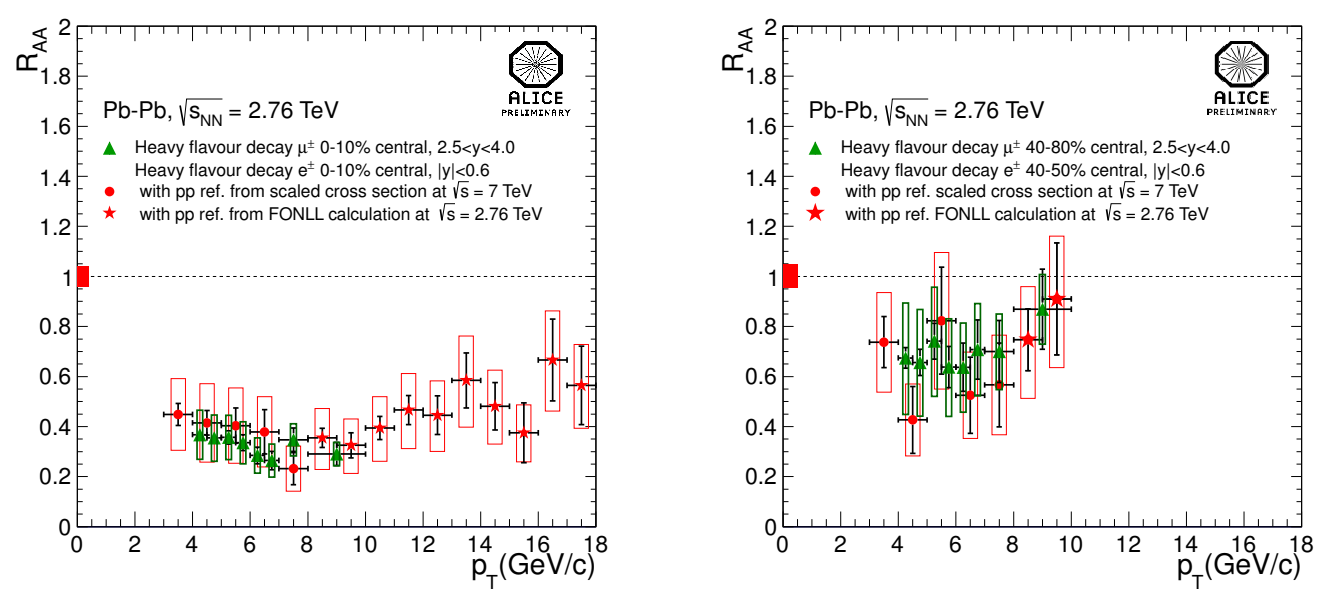

Figure 1. Nuclear modification factors of heavy-flavour decay electrons $(|y|<0.6)$ and muons $(2.5<y<4)$ as a function of $p_{\mathrm{T}}$ in most central (0-10\% centrality, left) and mid-central (40-50\% for electrons and 40-80\% centrality for muons, right) $\mathrm{Pb}-\mathrm{Pb}$ collisions.

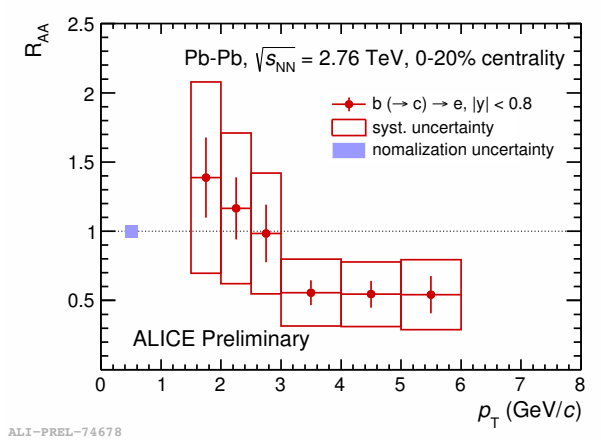

Figure 2. Nuclear modification factor of beauty-decay electrons $(|y|<0.8)$ as a function of $p_{\mathrm{T}}$ for the $20 \%$ most central class $\mathrm{Pb}-\mathrm{Pb}$ collisions.

Further information on the interaction of heavy quarks with the medium can be obtained via the measurement of the anisotropy in the azimuthal distribution of particle momenta in $\mathrm{Pb}-\mathrm{Pb}$ collisions. Experimentally, the azimuthal anisotropy has been studied using a Fourier expansion of the particle azimuthal distribution relative to the reaction plane, defined by:

$$
\frac{d N}{d \varphi}=N_{0}\left\{1+\sum_{n} 2 v_{n} \cos \left(n\left(\varphi-\Psi_{R . P .}\right)\right)\right\},
$$

where $N_{0}$ is a normalization constant, $\varphi$ is the azimuthal emission angle of particles, and $\Psi_{R . P}$. is the reaction plane. In particular the second harmonic coefficient $v_{2}$ of the expansion of the azimuthal distribution is called "elliptic flow". At low $p_{\mathrm{T}}$, it allows us to test if heavy quarks take part in the 
collective expansion of the medium, which is well described by hydrodynamical models. At high $p_{\mathrm{T}}$, the anisotropy reflects the path-length dependence of parton energy loss. Figure 3 shows the $v_{2}$ of heavy-flavour decay electrons $(|y|<0.7)$ and muons $(2.5<y<4)$ as a function of $p_{\mathrm{T}}$ in the $0-10 \%$ (left) and the $20-40 \%$ (right) centrality classes. We observe positive $v_{2}$ of heavy-flavour decay leptons with a significance of more than $3 \sigma$ in $2<p_{\mathrm{T}}<3 \mathrm{GeV} / \mathrm{c}$ in the $20-40 \%$ centrality class. This result suggests that charm quarks, which are the dominant source of heavy-flavour decay electrons at low $p_{\mathrm{T}}$, take part in the collective expansion of the medium. The measurements in the $0-10 \%$ and $20-40 \%$ centrality classes indicate that the strength of $v_{2}$ has a centrality dependence, i.e. $v_{2}$ is larger in the $20-40 \%$ central interval than in the $10 \%$ most central collisions. A comparison of the $v_{2}$ with heavyflavour decay muons at forward rapidity indicates that there is no significant rapidity dependence of the elliptic flow of heavy flavour particles.
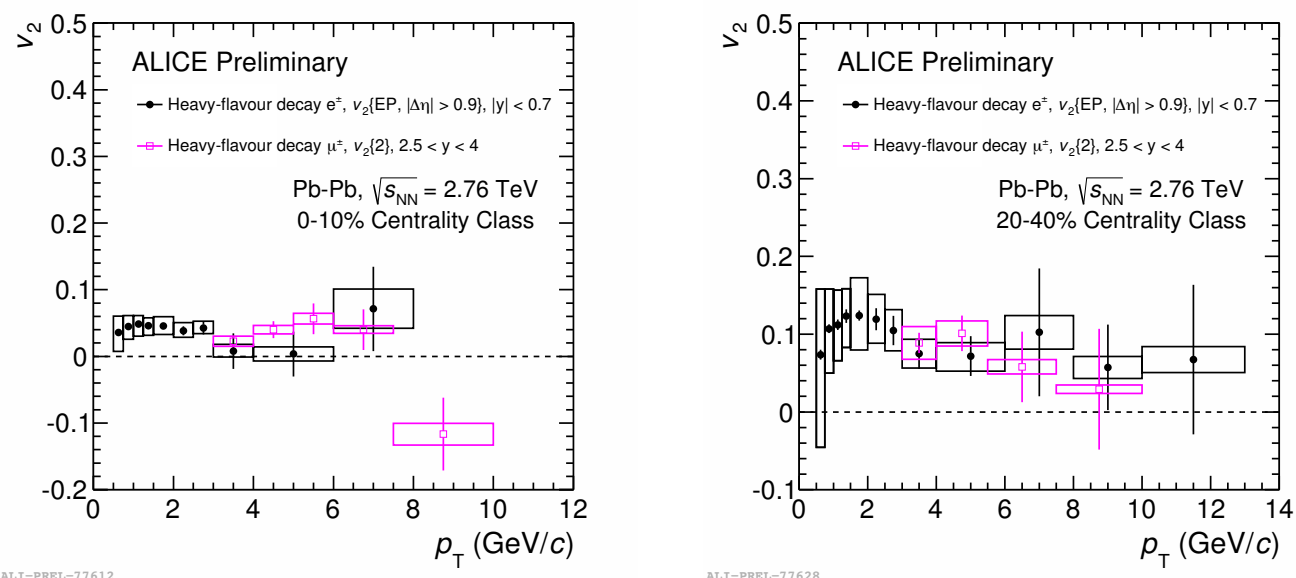

Figure 3. Elliptic flow of heavy-flavour decay electrons $(|y|<0.7)$ and muons $(2.5<y<4)$ in the $0-10 \%$ (left) and $20-40 \%$ (right) centrality classes in $\mathrm{Pb}-\mathrm{Pb}$ collisions.

In Fig. 4, the results for heavy-flavour decay electrons are compared with models that predict both $R_{\mathrm{AA}}$ and $v_{2}$ [14-17]. These models predict a strong suppression of heavy-flavour decay electrons at high $p_{\mathrm{T}}$ as it was observed in the experiment. On the other hand, the considered models have significantly different predictions for the $v_{2}$ of heavy-flavour decay electrons, especially at low $p_{\mathrm{T}}$. Thus a simultaneous description of $R_{\mathrm{AA}}$ and $v_{2}$ of electrons from heavy-flavour hadron decays can help to constrain the theoretical understanding of heavy quark interaction in the medium.

The influence of cold nuclear matter effects can be estimated by measuring heavy-flavour production in proton-nucleus collisions where an extended hot and dense medium medium is not expected to be formed. For this purpose, electrons and muons from heavy-flavour hadron decays were measured at $\sqrt{s_{\mathrm{NN}}}=5.02 \mathrm{TeV}$ in $\mathrm{p}-\mathrm{Pb}$ collisions with ALICE. In the left panel of Fig. 5, the transverse momentum dependence of the nuclear modification factors of electrons from decays of heavy-flavour $(\mathrm{b}, \mathrm{c} \rightarrow \mathrm{e})$ and beauty $(\mathrm{b} \rightarrow \mathrm{e})$ hadrons in $\mathrm{p}-\mathrm{Pb}$ collisions $\left(R_{\mathrm{pPb}}\right)$ are shown, and they are both consistent with unity within uncertainties. A comparison with a theoretical calculation with a pQCD calculation at FONLL for heavy-flavour production with the EPS09 parameterization of nuclear of PDFs [4] is shown in the right panel of Fig. 5, and theory and data are consistent within the uncertainties. $R_{\mathrm{pPb}}$ of muons from heavy-flavour hadron decays was measured at forward $\left(2.5<y_{\mathrm{cms}}<3.54\right.$, 

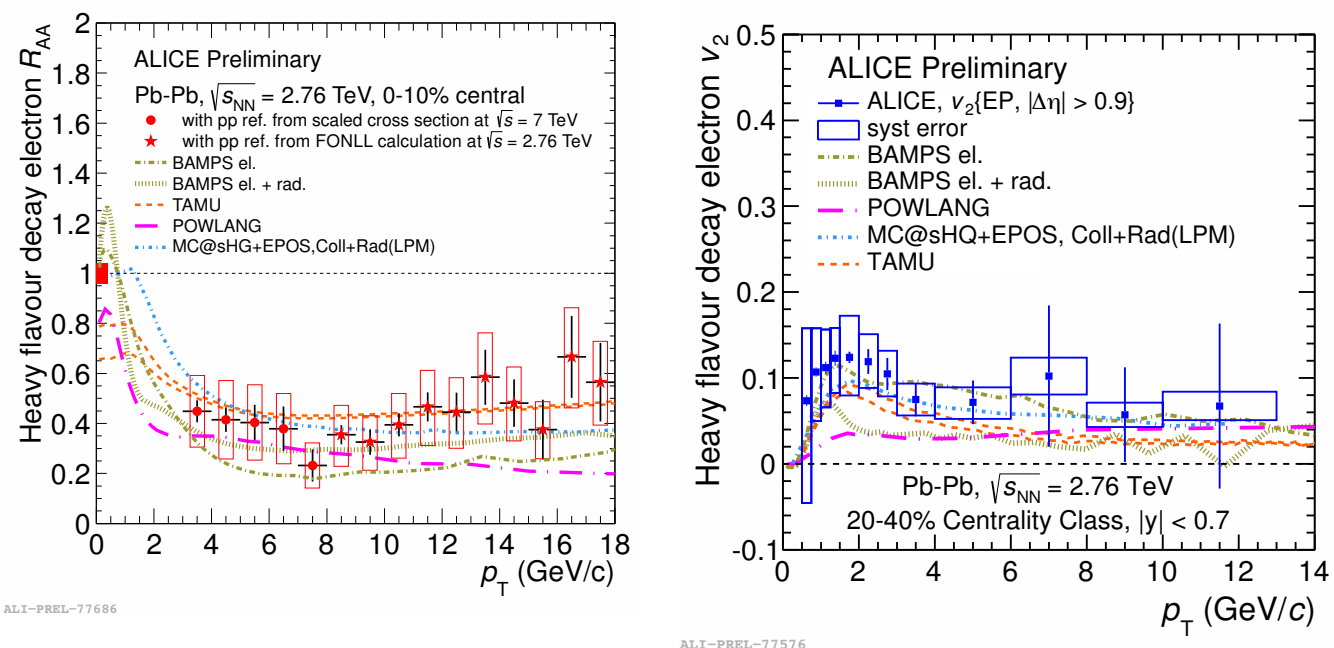

Figure 4. Comparison with theoretical calculations of the heavy-flavour decay electron $R_{A A}$ in the $10 \%$ most central $\mathrm{Pb}-\mathrm{Pb}$ collisions (left) and for $v_{2}$ in the $20-40 \%$ centrality classes (right).

proton-beam direction) and at backward rapidity $\left(-4<y_{\mathrm{cms}}<-2.96\right.$, Pb-beam direction). The results are shown in Fig. 6 and $R_{\mathrm{pPb}}$ at forward rapidity is consistent with unity, $R_{\mathrm{pPb}}$ at backward rapidity is consistent with unit for $p_{T}>4$ and slightly larger for $p_{T}<4$. Both observables are well described by calculations within uncertainties. From the results of electrons and muons in $\mathrm{p}-\mathrm{Pb}$ collisions, it can be concluded that the strong suppression of heavy-flavour production at intermediate and high $p_{\mathrm{T}}$ measured in $\mathrm{Pb}-\mathrm{Pb}$ collisions is mainly due to the interaction of heavy quarks with the hot and dense medium.
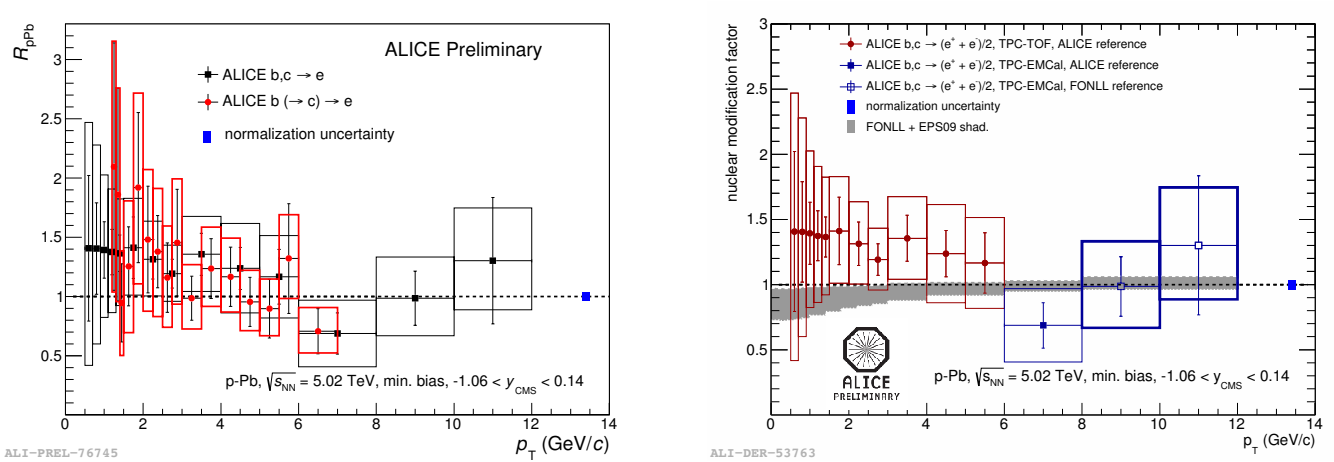

Figure 5. Nuclear modification factors of heavy-flavour decay electrons and beauty-decay electrons as a function of $p_{\mathrm{T}}$ in $\mathrm{p}-\mathrm{Pb}$ collisions (left) and in comparison with theoretical calculations (right).

In high-multiplicity $\mathrm{p}-\mathrm{Pb}$ collisions, a double-ridge structure has been observed in the angular $(\Delta \varphi, \Delta \eta)$ correlations between two charged particles. Such a structure is also found in the twoparticle correlations between heavy-flavour decay electrons and charged particles [18]. In Fig. 7 
(left), the correlation distribution in high-multiplicity (0-20\%) collisions after subtracting the correlation measured in low-multiplicity (60-100\%) collisions is shown. Long-range correlations are observed in pseudorapidity on the near-side and away-side in azimuthal direction. The subtraction of low-multiplicity events removes the correlation mainly due to jets, and the remaining correlation between heavy-flavour decay electrons and charged particles indicates that heavy-flavour production might be affected by gluon saturation in the initial state as predicted by the Color Glass Condensate (CGC) calculations and/or by a collectively expanding system in the final state as it is assumed in hydrodynamical model calculations.
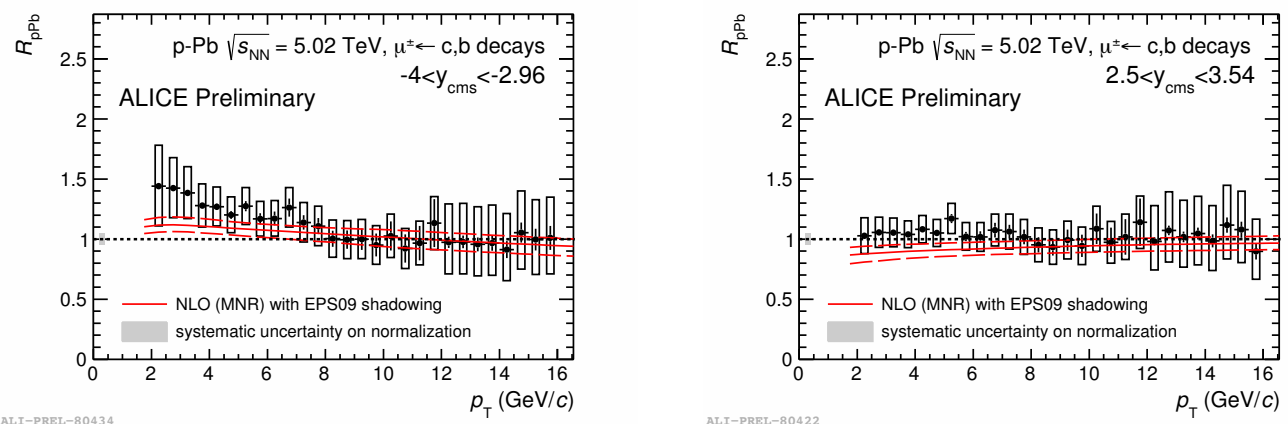

Figure 6. Nuclear modification factors of heavy-flavour decay muons in $\mathrm{p}-\mathrm{Pb}$ collisions at $-4<y_{\mathrm{cms}}<-2.96$ (left) and $2.5<y_{\mathrm{cms}}<3.54$ with theoretical calculations.
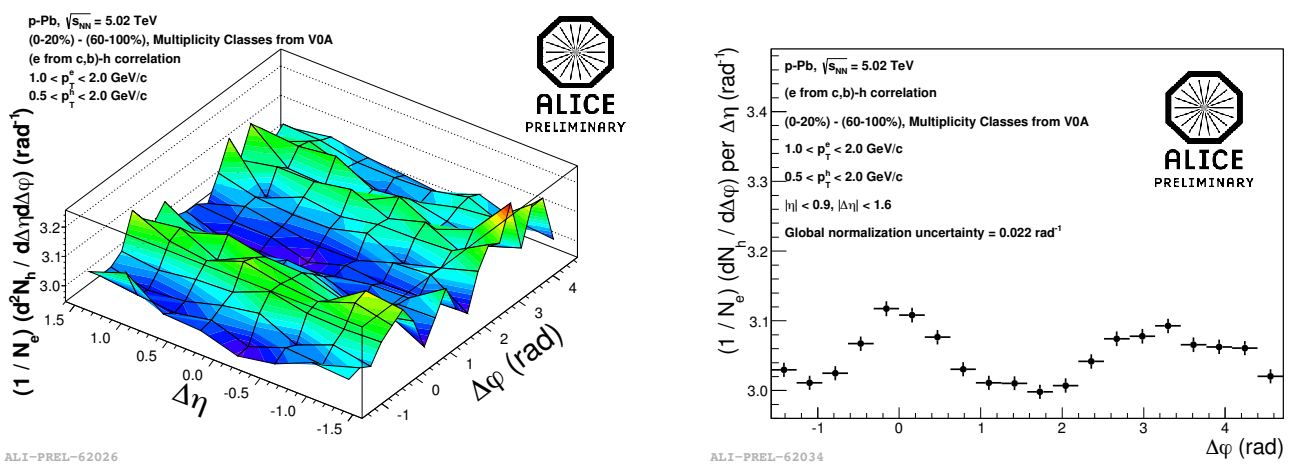

Figure 7. Difference between the two-particle angular $(\Delta \varphi, \Delta \eta)$ correlations between heavy-flavour decay electrons and hadrons in high (0-20\%) and low (60-100\%) multiplicity $\mathrm{p}-\mathrm{Pb}$ collisions (left) and projection of the difference on $\Delta \varphi$ axis (right).

\section{Summary}

The nuclear modification factor $\left(R_{\mathrm{AA}}\right)$ and elliptic flow $\left(v_{2}\right)$ of heavy-flavour decay electrons and muons have been measured in $\mathrm{Pb}-\mathrm{Pb}$ collisions at $\sqrt{s_{\mathrm{NN}}}=2.76 \mathrm{TeV}$ with ALICE at the LHC. A strong suppression of the yields at intermediate and high $p_{\mathrm{T}}$ in the $10 \%$ most central collisions and 
a positive $v_{2}$ in mid-central collisions have been observed. Measurements of $R_{A A}$ and $v_{2}$ of heavyflavour decay electrons at mid rapidity and muons at forward rapidity are compatible with each other indicating that there is no strong rapidity dependence in heavy quark in medium energy loss. In $\mathrm{p}-\mathrm{Pb}$ collisions, the nuclear modification factor $\left(R_{\mathrm{pPb}}\right)$ of electrons from decays of heavy-flavour $(\mathrm{b}, \mathrm{c} \rightarrow \mathrm{e})$ and beauty $(\mathrm{b} \rightarrow \mathrm{e})$ hadrons at mid-rapidity as well as that of heavy-flavour decay muons at forward and backward rapidity were measured. The results indicate that the cold nuclear matter effects on heavy-flavour production are small and that the strong suppression of the heavy-flavour yield observed at intermediate and high $p_{\mathrm{T}}$ in $\mathrm{Pb}-\mathrm{Pb}$ collisions is due to the presence of the hot and dense QCD medium. In $\mathrm{p}-\mathrm{Pb}$ collisions, we observed long-range correlations in pseudorapidity on the nearand away-side in azimuth between heavy-flavour decay electrons and hadrons, which are thought to be introduced by gluon saturation in the initial state in the CGC framework and/or hydrodynamical behavior in the final state.

\section{References}

[1] B. Abelev et al., [ALICE Coll.], JHEP 9 (2012) 112

[2] A. Adare et al., [PHENIX Coll.], Phys. Rev. Lett. 98 (2007) 172301.

[3] Yu. L. Dokshitzer and D. E. Kharzeev, Phys. Lett. B 519 (2001) 199.

[4] K. J. Eskola et al., JHEP 0904 (2009) 65

[5] H. Fuji and K. Watanabe, NPA 915 (2013) 1

[6] S. Chatrchyan et al., [CMS Coll.], Phys. Lett. B 718 (2013) 795.

[7] B. Abelev et al., [ALICE Coll.], Phys. Lett. B 719 (2013) 29.

[8] B. Abelev et al., [ALICE Coll.], Phys. Lett. B 726 (2013) 164.

[9] B. Abelev et al., [ALICE Coll.], Phys. Rev. C 88 (2013) 044909.

[10] B. Abelev et al., [ALICE Coll.], Phys. Lett. B 721 (2013) 13.

[11] R. Averbeck, N. Bastid, Z. Conesa del Valle, P. Crochet, A. Dainese, X Zhang, arXiv:1107.3243.

[12] M. Cacciari, S. Frixione, N. Houdeau, M. L. Mangano, P. Nason and G. Ridolfi, JHEP 1210 (2012) 137.

[13] B. Abelev et al., [ALICE Coll.], Phys. Rev. Lett. 109 (2012) 112301.

[14] J. Uphoff, O. Fochler, Z. Xu and C. Greiner, Phys. Lett. B 717 (2012) 430, arXiv:1310.3597

[15] M. He, R. J. Fries and R. Rapp, arXiv:1401.3817.

[16] M. Nahrgang, J. Aichelin, P. B. Gossiaux and K. Werner, Phys. Rev. C 89 (2014) 014905.

[17] W. M. Alberico et al., J. Phys. G 38 (2011) 124144.

[18] E. Pereira de Oliveria Filho [ALICE Coll.], arXiv:1404.3983. 